\title{
$\underline{\text { Axiological Scientific Realism and Methodological Prescription }}$
}

Timothy D. Lyons

Indiana University-Purdue University Indianapolis

\section{I: Meta-Hypotheses in the Scientific Realism Debate}

The contemporary scientific realism debate centers on two general kinds of meta-hypotheses, i.e., hypotheses about science, both of which are purported to be empirical. The first kind of metahypotheses are descriptive hypotheses regarding the nature of scientific inquiry. I will call these "Type-D meta-hypotheses." The second kind of meta-hypotheses are epistemological theories about what individuals (scientists or non-scientists) should / can justifiably believe about (successful) scientific theories. I will call the latter, "Type-E meta-hypotheses."

I will first identify three Type-Ds. The realist's most explicit and fundamental Type-D is axiological, a meta-hypothesis that purports to describe the aim of science: science seeks truth, including truth about unobservables. This meta-hypothesis is opposed by non-realists who favor an alternative. Van Fraassen, for instance, argues that science seeks empirical adequacy — and that truth about unobservables, though possibly attained on occasion, is not of concern to the scientific enterprise. Larry Laudan argues that, if a general aim is needed, it is that science seeks, not truth, but solutions to problems. Hence, central to the scientific realism debate are competing empirical descriptions, Type-D axiological meta-hypotheses, regarding the aim of science.

A second kind of Type-D meta-hypothesis of importance in the realism debate pertains more specifically to the nature and structure of scientific inference itself. In light of well-known descriptive concerns regarding the classical Type-D meta-hypotheses- e.g., enumerative-inductivism, hypothetico-deductivism - realists tend to favor an alternative that we can dub descriptive 
explanationism. This is the descriptive meta-hypothesis that much of the reasoning that occurs in science (as well as commonsense) is properly understood as an inference to the best explanation (e.g., Lipton, 2004) — one among many of its purported virtues being that it can account for the explanatory positing of unobservables. By contrast, non-realists, such as van Fraassen, who take truth about unobservables to be irrelevant to science's primary aim, have tended to de-emphasize the demand for explanation, construing that demand as merely pragmatic.

A third kind of Type-D meta-hypothesis, which, although related to the others, pertains most explicitly to the nature of changes in theoretical content that have occurred in the history of science. More specifically this third kind of Type-D purports to inform us of the extent to which the theoretical content of past theories and their successors has varied. While I will discuss each of these in more detail below, we can note that standard scientific realism embraces each of the following purportedly empirical Type-D meta-hypotheses:

- A Type-D axiological hypothesis: "science seeks truth."

- A Type-D methodological/inferential hypothesis: "scientists employ inference to the best explanation."

- A Type-D content-retention hypothesis: "successor theories retain as an approximation the deep theoretical content of their predecessors."

I will argue below that each of these requires considerable refinement. Nonetheless, notice that, in want of providing a comprehensive account of science, this collection of Type-Ds offers the realist a rather rich and empirically informative collection of meta-hypotheses. 
Notice also, however, that the primary thesis around which the realism debate has most centrally pivoted is not included in this list of Type-Ds. For that debate has almost entirely pivoted around the other kind of meta-hypothesis flagged above, epistemological or Type-E meta-hypotheses. Type-Es are meta-hypotheses about what individuals (scientists or non-scientists) should / can justifiably believe about (successful) scientific theories. A basic version of the Type-E meta-hypothesis embraced by standard scientific realism is, "We can justifiably believe that successful theories are approximately true (that the unobservable entities postulated within them exist, etc)." An example of a competing non-realist Type-E hypotheses is that "We can justifiably believe (only) that successful theories are empirically adequate (i.e. that what successful theories say about observables is true)."

The realism I advocate is a non-epistemic realism, one that endeavors to embrace only Type-Ds while bracketing concern with Type-Es (be they realist or non-realist). It treats Type-Ds the same way it treats scientific theories, not as objects of belief but as tools for inquiry, or more specifically, as tools to be deployed in the quest for truth. Fundamentally, it is axiological: while I agree with non-realists that the realist's epistemological meta-hypothesis (in its various forms) faces serious problems, taking a cue from Nicholas Rescher, I deny that this requires throwing out the realist's primary Type-D metahypothesis that science seeks truth. And here, due to the neglect of that axiological thesis, realists (including Rescher) have failed to give that axiological hypothesis substance. I've argued elsewhere (2005, forthcoming) that getting clear on just which subclass of true statements is sought in science (or just what is "the kind of truth science seeks"), and hence, articulating a more specific and informative version of the axiological meta-hypothesis, make an enormous difference to the realist attempt to provide an encompassing account of science. 
While the realism I advocate is fundamentally axiological, and I will discuss the axiological meta-hypothesis more in Section III, my primary concern in this paper will not be the specifics of the axiological hypothesis. Rather, my focus will be on the third kind of Type-D meta-hypothesis above, the content-retention hypothesis. I suggest it is with respect to the question of content-retention hypotheses that the debate over Type-Es has begun to inadvertently pay off. More specifically, I will argue, that the debate over Type-Es has been valuable and informative in the quest to describe and understand the nature of scientific inquiry, i.e., toward the development of a descriptive methodology. In Section III, I will explore the way in which my proposals bear on the second realist Type-D above, the explanationist hypothesis.

\section{II: The Methodological Value of the Debate over Retention Hypotheses in the Realism Debate}

In this section, I will argue that important methodological information can be extracted from the debate over the historical challenge to scientific realism. First, to clarify the nature of that non-realist challenge: Larry Laudan (1981) pits the history of science against standard scientific realism, in particular, against the realist's claimed connection between success and approximate truth. Taking reference to be a necessary condition for approximate truth, he lists successful theories whose central terms are such that, by present lights, they do not refer, e.g., 'phlogiston', 'caloric', a 'luminiferous ether', etc. The common interpretation of Laudan's argument is that it is a pessimistic meta-induction: from a list of past theories that were successful, but which cannot be approximately true, we infer the conclusion that our present successful theories are (likely) altogether false. I've argued elsewhere $(2002,2006)$, however, that this is ultimately a straw characterization of the historical argument 
(although Laudan does employ a meta-induction in his ((1977), p. 126)). I contend that Laudan's (1981) argument is properly understood as, not a meta-induction, but a meta-modus tollens. On this understanding, the successful theories that cannot be approximately true stand as falsifying instances of a fourth realist Type-D hypothesis, a semantic one, the one that standard realists in their Type-E claim we can justifiably believe: 'successful theories are approximately true.' Construing the historical threat as a meta-modus tollens, the non-realist's conclusion is not that contemporary scientific theories are false (as in the meta-induction); it is that the Type-D semantic meta-hypothesis that realists claim we can justifiably believe is false. Not only, then, cannot we not justifiably believe it, it cannot even be accepted as a 'fallible' or 'defeasible' conjecture. Although this is but one among a set of implications of the modus tollens (for a full discussion see my 2002), it suffices to make clear that the historical argument is not resolved by the common realist response of denying the legitimacy of an induction from past to present theories. Nonetheless, the modus tollens remains open to another general strategy employed to save realism from the pessimistic meta-induction: Seeking to eliminate the theories on Laudan's list, realists have revised the semantic Type-D they claim (in their Type-E) that we can justifiably believe.

For present purposes, I am setting aside the many challenges to epistemic realism, e.g., discussion of the no-miracles argument invoked to justify that belief; additional consequences of the modus tollens that bear on the latter; alternative explanations for success; the fact that surviving the modus tollens does nothing to imply a further capacity for explaining success; problems of underdetermination, etc. In fact, the goal of this section (and this paper a whole) is not to challenge epistemic realism; the goal is rather to extract from the debate over Type-Es one particular aspect of that debate that, I am arguing, is informative with respect to the nature of scientific inquiry. To make 
this clear: in response to the historical data, such as those offered by Laudan, realists have proposed a series of Type-Es-e.g. "we can justifiably believe that successful scientific theories are approximately true". Each of these asserts justification for believing a semantic Type-D-e.g. "successful theories are approximately true". In turn, these semantic Type-Ds entail a syntactic Type-D, a content-retention meta-hypothesis - e.g. successors retain at least an approximation of the theoretical content of their successful predecessors. What is of particular concern for present purposes is that, in the course of modifying their Type-Es, realists have modified their Type-D syntactic retention meta-hypothesis. Because it is with the latter that the historical debate can benefit our understanding of scientific inquiry, ${ }^{1}$ my goal in what follows is to survey only the most prominent syntactic Type-D retention hypotheses (bracketing off both the Type-Es, and the specific semantic Type-D that the Type-Es claim justifiably believe). My survey of these Type-D syntactic retention meta-hypotheses will be roughly structured in order of decreasing content (and increasing fortitude against the modus tollens).

Returning now to standard scientific realism, its retentionist meta-hypothesis is "successors retain or at least approximate the theoretical content of their successful predecessors." By way of the modus tollens, Laudan's (1981) data refutes this meta-hypothesis, prompting new variations. Explicitly in response to Laudan's list, a number of realists make an appeal to novel predictive success. Again, of concern here is the entailed retention hypothesis, "successors retain or at least approximate the theoretical content of their predecessors when the latter achieve novel predictive successes." Going beyond Laudan's text, this retention hypothesis also faces counterinstances, as pointed out by Worrall (1989) and others. The now standard example here is the successful novel prediction of the white spot

\footnotetext{
${ }^{1}$ Of course, the question of content retention has a long history that has been inherited and advanced within the scientific realism debate. While Laudan explicitly rejects Kuhnian incommensurability, he denies what he takes to be the exaggerated continuity to which standard realism commits itself.
} 
derived by Poisson from Fresnel's ether theory. (For others see my 2002.) Mindful of some such examples, Stathis Psillos (1999) provides what is arguably the most sophisticated variant of a realism that embraces the attribution of approximate truth. Adding to a demand for novel success, the core element of Psillos's strategy is to attribute approximate truth, not to theories as a whole, but only to those constituents that were genuinely deployed in, "responsible for," particular novel successes. Again, of concern here is the deployment realist's retention hypothesis: "successors retain or at least approximate the theoretical content of their predecessor's constituents that were responsible for particular novel successes." Invoking the modus tollens, and exploring the reasoning employed by Kepler, Newton, Leverrier and Adams, I've detailed numerous counterinstances to this hypothesis in my (2006) (pointing to others in my (2002)). Although each of the following constituents was genuinely deployed in novel successes, each is such that successor theories did not retain even an approximation of its theoretical content:

- the sun is a divine being and/or the center of the universe; (Kepler)

- the natural state of the planets is rest;

- there is a non-attractive emanation coming from the sun that pushes the planets forward in their paths;

- the planets have an inclination to be at rest, and to thereby resist the solar push, and this contributes to their slowing speed when more distant from the sun;

- the force that pushes the planets is a "directive" magnetic force;

- there exists only a single planet and a sun in the universe (Newton);

- each body possesses an innate force, which, without impediment, propels it in a straight line infinitely; 
- between any two bodies there exists an instantaneous action-at-a-distance attractive force;

- the planet just beyond Uranus has a mass of 35.7 earth masses (Leverrier)/ 50 earth masses (Adams);

- that planet has an eccentricity .10761 (Leverrier)/.120615 (Adams)

- the longitude of that planet's perihelion is $284^{\circ}, 45^{\prime}$ (Leverrier) $/ 299^{\circ}, 11^{\prime}$ (Adams), etc.

In the face of these counterinstances, a natural (though anachronistic) step is to sidestep the attribution of approximate truth to theoretical constituents, emphasizing instead only the existence of entities that are subject to manipulation (Hacking, 1984). The relevant Type-D retention hypothesis is (something like) "successor theories retain those entities that were, not only posited in predecessor theories, but that have also been subject to manipulation." The ubiquitous objection is that, without some kind of theory, this form of realism lacks content: it will be theory that informs us of an entity's properties and whether it is being manipulated; and just how entities can be distinguished from, or require only lowlevel, theories remains unclear. (This point accords with Worrall's critique (EPSA, 2009).)

Accordingly, it remains unclear that one's claim to be manipulating electrons does not depend on the fact that it is theory that tells us what electrons are. Or, more directly in regard to the historical argument, it remains unclear why, from the context of phlogiston theory, the deliberate directing of even a flame does not count as manipulating phlogiston ("the stuff of flames"), and why, from the context of caloric theory, the deliberate transfer of heat does not constitute the manipulation of caloric, etc. The vagueness of, and consequent difficulty in testing, entity realism may account for the shortage of case studies (but see, for instance, Carrier, 1993). (Here is one arena in particular calling for greater articulation and historical exploration.) Insofar as theory is required to inform us of the properties that 
are to be attributed to entities, and provided we understand the historical argument as a modus tollens, the extent to which the entity realist retention hypothesis fares better than the others remains unclear.

Perhaps the most prevalent contemporary contender for an alternative to standard scientific realism is epistemic structural realism, which, in contrast with entity realism, was introduced by Worrall (1987) with specific concern for the historical argument. The relevant retentionist hypothesis states essentially that "successor theories retain or at least approximate the mathematical structure of those predecessors that have achieved novel success." Against the meta-modus tollens, it appears that structural realism fares better than the retention meta-hypotheses considered above. (One concern is as follows: although structural approximation may be such that it can obtain at the kind of depths described within, say, quantum chromodynamics or M-Theory, it is unclear why it cannot equally obtain at levels as shallow as the observed data. And, insofar as any successor that approximates its predecessor's success also approximates that predecessor's shallow structure, structural realism has at least the potential to be protected from nearly any purported counterinstance.) Continuing our very brief focus on, and survey of, retention hypotheses, while Worrall looks to Poincare, Martin Carrier, looking to Duhem's notion of a "natural classification," advocates a classificatory or taxonomical realism. (Because I think we can reasonably talk about structural components of a theory that are not merely classificatory, I will take the liberty of treating this as a less demanding thesis than structural realism.) The retention hypothesis is that "successor theories retain the classification or taxonomy of those predecessors that have achieved novel success." While each of the above retention hypotheses may need clarification, and each calls for further testing, I do think that each holds the potential for both. 


\section{III: A Return to the Realist's Explanationist and Axiological Type-Ds}

I began this paper by discussing three kinds of descriptive, Type-D, meta-hypotheses. Beyond the content-retention Type-D, another of these is the realist's explanationist Type-D. Although realists often invoke the meta-hypothesis that much of scientific reasoning can be understood as an inference to the best explanation, realists also often concede that it remains unclear just how this mode of inference restricts our theorizing. In this section I will endeavor to illustrate how a framework based on the retentionist and axiological Type-D's can be of significant value in the articulation of the explanationist Type-D meta-hypothesis.

First, however, we need to follow the path traced in the last section. Note that, insofar as each of the above Type-Es and their respective entailed Type-D retention hypotheses stand as a response to the data, each of the Type-D retention hypotheses, in effect, offers greater allowance for non-retention in theorizing. Even the standard scientific realist's retention hypothesis effectively allows that successors only retain their predecessors as an approximation. And these allowances increase as we move through our series of retention hypotheses. Arriving at the classificatory realist's retention hypothesis, we see that it allows that successors do not retain or even approximate theoretical posits, even those deployed in novel predictions; it allows replacement of even manipulated entities; and it may even permit the non-retention of structural elements that are non-classificatory. If the empirical evidence turns out to bear on the sequence of retention hypotheses, as that sequence was unfolded in the last section, then, in terms of content at least, scientific theorizing is afforded a significant and potentially surprising degree of what might be appropriately dubbed creative license. In fact, the following is a bold statement on permissibility or license in scientific theorizing that can be drawn from the path traced in Section II: given the historical data, the theoretical content of successors has not been required to retain or even 
approximate the theoretical content of their predecessors; and this is so, even if the predecessor theory / constituent-set had achieved novel successes, had been deployed in the derivation of those novel successes, was such that its entities were taken to have been manipulated, etc.

Potentially providing information of which we may not have been aware, this statement is informative with respect to our efforts to understand scientific inference. However, because, it appears that, at least when it comes to content, the historical data support creative license, this information ultimately decreases the restrictiveness of our model of inference. On the one hand, although we may seek to identify greater restrictions imposed on our theorizing, the historical data may now have pushed us in the other direction; inference to the best explanation may well not be as restrained as we may have hoped.

On the other hand, however, the historical data do not suggest that theorizing is wholly unrestricted. That is, upon more careful consideration of the (albeit preliminary) data, the data also suggest ways in which creative license may be tempered. I suggest here that, given present and future data, meta-hypotheses that express flexibility in theorizing can, in principle, be ranked from higher priority (the classificatory realist's retentionist hypothesis) to lower priority (the standard scientific realist's retentionist hypothesis), in inverse relation to the quantity of counterinstances discerned in the literature. That is, the historical data when unfolded as above provide an opportunity to develop a distribution of retention-priorities. (I will illustrate this below.)

Beyond such a priority ranking, present and future data may offer further refinement regarding the "distance" between the priorities, or the degree to which such priorities differ. It may turn out, for instance, that the second retention-priority (say, that successors retain as an approximation the 
mathematical structure described by their predecessors) is a vastly greater retention-priority than the third (say, that successors retain the predecessor's existential posits that are subject to manipulation). Hence, on one hand, in terms of creative license and non-retention, the data appear to be such that they render our model of inference less restrictive; on the other hand, we see that a more careful analysis and, ultimately, a priority ranking can reveal an increase in the restrictions on inference. Both modes of evaluation offer a stronger empirical foundation for our meta-hypotheses about science.

Yet, in fact, a greater and even more tangible collection of restrictions is available given other realist Type-Ds. For, of course, our attempt to account for scientific inference is not limited to considerations regarding content-retention. We can also add, crucially, historically informed Type-Ds regarding the demand for increases in, and the retention of, specific theoretical virtues. This returns us full circle to the first kind of Type-D meta-hypothesis I discussed in Section I. As mentioned there, I advocate a refined axiological realist meta-hypothesis, one that endeavors to specify the subclass of true claims sought within science. After briefly indicating the nature of that postulate, I will indicate a way in which it is informative with respect to theoretical virtues. (While the details of my hypothesis and its relation to theoretical virtues can be found in my (2005), I provide only a snapshot here to illustrate its promise toward developing an empirically informed realist conception of science.)

The general idea of that meta-hypothesis is that science seeks to increase the manifest truth the MT statements - of its theory complexes. MT statements are those statements that are not merely true but made manifest as true; that is, they are statements whose truth is concretized in, logically pushed to, reports documenting specific experiences. (It is important to emphasize that the manifestation here is not epistemic; it is not that the truth of MT statements is made manifest to us; rather the truth of MT statements is deductively made manifest in, pushed to and made concrete in, 
other statements, specifically, again, reports documenting specific experiences.) Regarding what is sought, then, this postulate constitutes a shift from "truth" to a subclass of true statements. Regarding the particular statements whose truth is sought, the postulate includes a shift from theories to the more encompassing collection of statements included in theory complexes/systems. (And while realist truth is not contingent on the system of statements to which a statement is conjoined, the manifestation of a statement's truth is so contingent.) The postulate also marks a shift from endeavoring to simply attain the truth to endeavoring to increase the quantity of true statements in the specified subclass. Moreover, with "increase" explicitly included in the postulated goal, evaluation is explicitly comparative: theory complex against theory complex, e.g., successor complex against predecessor. Most importantly, I've shown in my (2005) that the actual achievement of this state, an increase in the MT statements of a theory complex, requires the achievement of a set of theoretical desiderata: namely, an increase in empirical accuracy and consistency, and an increase in, or at least the retention of, breadth of scope, testability, and number of forms of simplicity. Like the content-retention hypotheses of concern in Section II, these desiderata can be understood in terms of syntactic relations; however, quite unlike those content-retention meta-hypotheses, these syntactic relations do not pertain to, or depend on, content relations between systems.

Of course, taken alone, the fact that these virtues are required in order to achieve the aim I've posited is not meant to provide evidence that descriptively science pursues these desiderata. Rather, the relevant empirical evidence is appealed to by authors as diverse as Kuhn, McMullin, Thagard, Lipton, as well as Laudan and van Fraassen. In contrast with Type-D retentionist hypotheses there is a considerable degree of agreement that these (and such) desiderata are maintained across successor and predecessor. I suggest that, my particular version of the realist's axiological meta-hypothesis offers 
both an explanation, and crucially, a justification for the pursuit of these otherwise potentially disparate theoretical virtues: if we don't have these necessary conditions for our primary goal, an increase in manifest truth, we know we don't have what we're after. I've argued that, not only does this meta-hypothesis dramatically improve the realist's ability to account for what is going on in science, it provides a better account than its non-realist competitors, such as Laudan's problem-solving model and van Fraassen's constructive empiricist model.

Having now (all too briefly) introduced my axiological hypothesis, I can now combine these points with our above considerations on ranking retention hypotheses. In order to make clear the form that a historically informed complex of Type-D retention meta-hypotheses might take - that is, in order to illustrate the manner in which a distribution of retention-priorities can be offered - consider the following sample-ranking (Sample A). Here, for the sake of illustration and simplification, we can assume that, in light of the data, the order of proposals stands as they were unfolded in Section II: the first set of proposals (e.g. standard scientific realism) have been found to have a greater number of counterinstances than the later proposals (e.g., classificatory realism). As noted above, given additional data, such a ranking could be advanced further to specify degrees of priority. While this ranking (Sample A) provides some help in restricting the degree of creative license flagged earlier, I can now add (in Sample B) the crucial restrictions derivable from my axiological realist meta-hypothesis, namely the theoretical virtues noted above:

Sample A: Content-Retention Priorities: Going from higher to lower, the priorities for retaining a successful predecessor's content in a successor are: 
- First Priority: the predecessor's classification of phenomena (in accord with the proposal by, e.g., Carrier)

- Second Priority: the predecessor's mathematical structure (or approximation thereof) (in accord with the proposal by, e.g., Worrall)

- Third Priority: the predecessor's existential posits that are subject to manipulation (in accord with the proposal by, e.g, Hacking)

- Fourth Priority: the predecessor's constituents (or approximation of the constituents) that have been deployed in novel successes (in accord with the proposal by, e.g, Psillos)

- Fifth Priority: the predecessor's non-deployed constituents (in accord with the proposal by, e.g., the standard scientific realist)

- $n$th Priority) ...

Sample B: Theoretical Virtues: Despite such license in terms of predecessor content, successor complexes, when compared to their predecessor complex, achieve the following theoretical desiderata:

- an increase in empirical accuracy and consistency and

- an increase in, or at least the retention of, breadth of scope, testability, simplicity, etc. As I hope is clear, my emphasis here is not so much on the particulars of what I'm proposing but on the prospects for the broader project of developing a rich and encompassing realist account of science. As noted in Section I, the realism I favor is non-epistemic: like scientific theories, Type-Ds are not treated as objects of belief, but as tools for further inquiry. Accordingly, and importantly, provided that we can develop such a collection of empirical meta-hypotheses, such a framework can be empirically informative; and it can be tested, as a set, against present and forthcoming data on intra-system 
relations, and against competing (e.g, non-realist) frameworks. Although this is only a preliminary sketch, to be substantially refined given further research, I suggest that, by drawing on the details of the realism debate, and digging deeper into the questions that have arisen in the interplay between realist Type-Ds and the historical data, the prospects for developing an informative, empirical, and truth-based account of science look promising. Finally, given the potentially informative nature of such a framework, I suggest that much of what is involved in, say, "inference to the best explanation," can now be understood, not as being added to, but, crucially, as following from the axiological and retentionist Type-D framework. I suggest that the latter holds significant promise for advancing and unifying our understanding of scientific inquiry.

I close with two comments. The first is a proposal that the framework, once sufficiently tested against the data and against competing frameworks, can be made prescriptive, and tested as such. The second is simply a plea to bring on the case studies - in want of developing both components of the framework, the Type-D retentionist rankings and their, potentially more primary, axiological conjuncts.

\section{$\underline{\text { References }}$}

Carrier, Martin. (1991), “What is Wrong with the Miracle Argument?' Studies in History and Philosophy of Science. vol. 22, pp. 23-36.

Carrier, Martin. (1993), "What is Right with the Miracle Argument: Establishing a Taxonomy of Natural Kinds,” Studies in History and Philosophy of Science. vol. 24, no.3, pp. 391-409. 
Hacking, Ian. (1983), Representing and Intervening. Cambridge : Cambridge University Press.

Lipton, Peter (2004), Inference to the Best Explanation, 2nd Edition, London: Routledge.

Lyons T. D. (2002), "Scientific Realism and the Pessimistic Meta-Modus Tollens," Recent Themes in Philosophy of Science: Scientific Realism and Commonsense, in S. Clarke and T.D. Lyons (eds), Dordrecht: Kluwer, pp. 63-90.

Lyons T.D. (2005) "Toward a Purely Axiological Scientific Realism" Erkenntnis 63:167-204

Lyons T.D. (2006): "Scientific Realism and the Stratagema de Divide et Impera," The British Journal for the Philosophy of Science, 57, pp. 537-560.

Laudan, L. (1977), Progress and its Problems, Oxford University Press, Oxford.

Laudan, Larry. (1981) “A Confutation of Convergent Realism,” Philosophy of Science, 48, pp. 19-49.

Psillos, Stathis. (1999) Scientific Realism: How Science Tracks Truth. London : Routledge.

Van Fraassen, Bas C. (1980) The Scientific Image. Oxford : Oxford University Press.

Worrall, John. (1989a) "Structural Realism : The Best of Both Worlds?” Dialectica, 43, 99-124. 